\title{
Simultaneously estimating food web complexity and structure with uncertainty
}

\author{
Anubhav Gupta ${ }^{1}$ and Owen Petchey ${ }^{1}$ \\ ${ }^{1}$ University of Zurich
}

May 23, 2021

\begin{abstract}
1) Food web models explain and predict the trophic interactions in a food web, and they can infer missing interactions among the organisms. The allometric diet breadth model (ADBM) is a food web model based on the foraging theory. In the ADBM the foraging parameters are allometrically scaled to body sizes of predators and prey. In Petchey et al. (2008), the parameterisation of the ADBM had two limitations: (a) the model parameters were point estimates, and (b) food web connectance was not estimated. 2) The novelty of our current approach is: (a) we consider multiple predictions from the ADBM by parameterising it with approximate Bayesian computation, to estimate parameter distributions and not point estimates. (b) Connectance emerges from the parameterisation, by measuring model fit using the true skill statistic, which takes into account prediction of both the presences and absences of links. 3) We fit the ADBM using approximate Bayesian computation to 16 observed food webs from a wide variety of ecosystems. Connectance was consistently overestimated in the new parameterisation method. In some of the food webs, considerable variation in estimated parameter distributions occurred, and resulted in considerable variation (i.e. uncertainty) in predicted food web structure. 4) We conclude that the observed food web data is likely missing some trophic links that do actually occur, and that the ADBM likely predicts some links that do not exist. The latter could be addressed by accounting in the ADBM for additional traits other than body size. Further work could also address the significance of uncertainty in parameter estimates for predicted food web responses to environmental change.
\end{abstract}

\section{Hosted file}

ms_C1.pdf available at https://authorea.com/users/415230/articles/523132-simultaneouslyestimating-food-web-complexity-and-structure-with-uncertainty 